\title{
LINKAGE MAPPING FROM PAIR-WISE RECOMBINATION DATA
}

\author{
J. M. LALOUEL* \\ Population Genetics Laboratory, University of Hawaii, Honolulu, 96822
}

Received 17.viii.76

\begin{abstract}
SUMMARY
The problem of obtaining a genetic map of a linkage group from pair-wise recombination data is considered. A non-parametric approach is proposed, that does not require the definition of a mapping function, computation of coefficients of coincidence, nor knowledge of map length or sex differences in recombination. An application to Bridges and Morgan's (1923) data on chromosome 3 of Drosophila melanogaster is presented.
\end{abstract}

\section{INTRODUGTION}

WE here propose a general solution to the problem of linear mapping of a linkage group from pair-wise recombination data. We are concerned with the following experimental situation: tests for detection of linkage have proven $n$ loci $L_{1}, L_{2}, \ldots, L_{n}$ to belong to the same linkage group; appropriate experiments have been carried out to estimate the pair-wise recombination fraction $\theta_{i j}$ between loci $L_{i}$ and $L_{j}$, although generally not for all the $n(n-1) / 2$ possible pairs. One would then like to give a linear map of this linkage group, the order and spacing of the $n$ loci being inferred from these recombination data. The present approach departs from others in at least two important respects: it does not require the assumption of a mapping function, nor does it compute coincidence coefficients. The theoretical justification of this approach requires the recall of some fundamental definitions and the consideration of our state of knowledge about the mechanism of recombination. A method for solution will then be presented, followed by illustrative examples with data on Drosophila melanogaster.

\section{REGOMBINATION FRAGTION AND MAP DistANGE}

(i) Some definitions

The recombination fraction $\theta_{i j}$ between loci $L_{i}$ and $L_{j}$, located on the same chromosome, is the probability of occurrence of an odd number of points of exchange on the same strand in the interval between these two loci (in the absence of chromatid interference, which we shall consider negligible). Because of the two phenomena of multiple crossing-over and positive interference - the occurrence of one cross-over tending to inhibit the occurrence of a second cross-over in its neighbourhood-recombination is not additive over

PGL paper no. 153. This work was supported by Grants GM 17173 and GM 23021 from the U.S. National Institutes of Health and by Grant $1-475$ from the National Foundation.

* Author is on leave from INSERM, Institut de Progénèse, and Institut National d'Etudes Demographiques, Paris. 
contiguous segments; that is, for three loci $L_{i}, L_{j}, L_{k}$ located in that order on the same chromosome, we have (e.g. see Owen, 1950):

$$
\theta_{i k}=\theta_{i j}+\theta_{j k}-2 z_{i j k}
$$

$z_{i j k}$ being the probability of simultaneous recombination in both segments $L_{i}, L_{j}$ and $L_{j}, L_{k}$. If there is no interference, then $z_{i j k}=\theta_{i j} \theta_{j k}$; with positive interference, $z_{i j k}<\theta_{i j} \theta_{j k}$. The coincidence ratio $C_{i j k}=z_{i j k} / \theta_{i j} \theta_{j k}$ is useful in order to investigate the intensity of interference among various chromosomes or segments of chromosome of a given species. Eq. (1) shows that $\theta_{i k} \leqq \theta_{i j}+\theta_{j k}$, with equality holding exactly for complete interference $\left(C_{i j k}=0\right)$, and approximately for segments small enough so as to make $z_{i j k}$ negligible.

The recognition of this has led to the definition of the map distance $w_{i j}$ between $L_{i}, L_{j}$ as the average number of points of exchange per strand between $L_{i}$ and $L_{j}$ over a large number of meioses. For three loci $L_{i}, L_{j}$, $L_{k}$ in that order, we have:

$$
w_{i k}=w_{i j}+w_{j k}
$$

whether there is interference or not, since the expectation of the sum of two randorn variables is equal to the sum of their expectations, whether they are independent or not. Therefore $w$ is a natural index of the total amount of crossing-over that may occur in a segment, and in the following map distance will be understood in that sense.

But whereas recombination can be observed, map distance can only be inferred from recombination. Hence the general approach to the mapping problem consists of estimating recombination fractions from appropriately designed crosses, and then inferring map distances through a mapping function embodying assumptions about the phenomenon of genetic crossover.

Various experimental designs and estimation procedures for the recombination fraction have been developed (e.g., Bailey, 1961; Robinson, 1971). The true value of the recombination fraction $\theta_{i j}$ between two loci $L_{i}, L_{j}$ is estimated by $y_{i j}$, the standard error of this estimate being denoted by $s_{i j}$.

\section{(ii) The problem of the mapping function}

As for the derivation of a mapping function, it has received much attention. The most widely used functions (Haldane, 1919; Kosambi, 1944; Carter and Falconer, 1951), attempting to take into account simultaneously the two phenomena of multiple crossing-over and interference, express map distance as a monotonically increasing function of recombination $\theta_{i j}$. As noted by Owen (1950), to express map distance solcly as a function of recombination, without considering the location of the interval under study, is equivalent to assuming uniformity of interference along the chromosome. However, various studies with Drosophila or the house mouse have shown that coincidence varies not only according to the length of the segments under study, but also according to their position on the chromosome. A better solution of the mapping problem would associate, to two equal recombination fractions $\theta_{i j}=\theta_{k l}$ for two segments of a chromosome, map distances $w_{i j}$ and $w_{k l}$ generally different. This requires map distance to be 
function of both recombination and location of the segment on the chromosome. Attempts have been made in this direction by Fisher, Lyon and Owen (1947), and Owen (1949, 1950). Their derivations, under Mather's theory of a serial process of chiasma formation (Mather, 1936, 1937, 1938), rest upon assumptions about chiasma distribution and an interference metric. Despite the interesting analytical insight they provide about recombination, their usefulness for the mapping problem is limited for two main reasons: the various assumptions required about chiasma formation, and the necessity of cytological data on chiasma distribution. Mather's theory still lacks clear enough experimental confirmation to be universally accepted. In effect, the only exhaustive study of chiasma distribution where Mather's theory was critically examined, carried out by Henderson (1963) on the locust Schistocerca gregaria, at least suggests a much more complex phenomenon, with chiasma formation possibly starting from the two opposite ends of the acrocentric chromosomes of this species. More generally, in the present state of cytological knowledge, the process of chiasma formation seems far too complex and variable among species, as well as among different chromosomes of a given species, for an exact mapping function to be derived from genetic data and theoretical assumptions alone. The phenomena of chiasma localisation, insufficient knowledge about the mechanism of crossing-over, its possible genetic regulation, and the distribution of the genetic material along the chromosome, make this approach impracticable for the mapping problem, whether or not cytological observations on chiasma distributions are available (e.g., John and Lewis, 1965; Henderson, 1969).

All that is needed for the mapping problem is a method that can make use of the non-additivity of recombination between adjacent segments, as shown in formula (1), to yield a genetic map allowing for variable interference. Then interference can be considered as a purely genetic phenomenon, and need not be estimated explicitly. Moreover, data or assumptions about chiasma distribution are not necessary, so that the mapping can be carried from genetic data only, with a minimum of unverified assumptions. Particularly, no explicit mapping function will be needed. Such a method will be proposed in part 3 .

\section{(iii) Chromatid interference}

Explicit reference to the phenomenon of chiasma interference, or nonrandom occurrence of multiple cross-overs, has been made earlier. There is another phenomenon, namely that of chromatid interference, that may complicate the problem of deriving a mapping function. Chromatid interference is a non-random relationship between those two strands which cross over at a second chiasma with respect to the two crossing over at the first. Cionsideration of chromatid interference is necessary to explain observations of recombination fractions greater than $0 \cdot 50$. The existence of this phenomenon is questionable, and it has been generally disregarded with reference to mapping. In the following, it will not be considered explicitly, as no mapping function is used and recombination fractions greater or equal to 0.50 will be neglected. That this is a negligible loss of information is clear from the fact that, although such values provide information about separation, they do not contain information about distance of separation. More details about chromatid interference may be found in Owen (1950). 


\section{(iv) Sex differences in recombination}

It is widely recognised that recombination may differ significantly according to sex in various species. There may not be any general rule to account for this difference. Haldane's rule (Haldane, 1922), that, where differences in crossing-over are found in the two sexes, it is the heterogametic sex in which crossing-over is lowered in frequency or absent, suffers notable exceptions (Fisher and Landauer, 1953; Sokoloff, 1964; Dunn and Bennett, 1967). Although based on Mather's controversial theory of chiasma formation, an interesting aspect of a paper by Carter (1954) was to show that, even if chiasma interfercnce tended to be lower in females than males, with the consequence of generally greater frequency of crossing-over in females, one could still expect some regions where recombination would be greater among males.

However, sex differences in recombination may have a far more complex explanation. Differences in chiasma localisation among sexes (Henderson, 1961; Watson and Callan, 1963), differences in the duration of chromosome pairing (John and Lewis, 1965), and other factors all point to the likelihood of differences in chiasma distribution among sexes. Hence, even though physical arrangement of genes is identical for the two sexes, this is not so as far as genetic distances are conccrned, so that mapping should be carried out separately for each sex.

\section{MAPPING FROM REcombination DATA}

Let us restate the mapping problem more specifically: for $n$ loci of a linkage group, recombination estimates $\left\{y_{i j}\right\}$ and their standard-errors $\left\{s_{i j}\right\}$ are available pair-wise, although generally not for all possible pairs. From these, we want to estimate the coordinates $\left\{x_{i}\right\}$ of the loci on a unidimensional scale such that map distances $\left\{d_{i j}\right\}$, as previously defined, are given by

$$
d_{i j}=\left|x_{i}-x_{j}\right|
$$

Although we shall consider here a method which does not necessitate an explicit mapping function $w=f(\theta)$ satisfying (2), it is worth mentioning that even if such a function were to exist and were known, this would not solve completely the mapping problem, because estimated map distances $\left\{\hat{w}_{i j}\right\}=\left\{f\left(y_{i j}\right)\right\}$, due to errors of estimation, would not satisfy (2) exactly: a procedure to yield the coordinates $\left\{x_{i}\right\}$ would be required; in particular, the method of maximum likelihood would require specification of the distribution of $\hat{w}$.

\section{(i) The recombination fraction is itself a useful metric}

Let us assume that, for three loci $L_{i}, L_{j}, L_{k}$ in that order on the same chromosome, we have:

$$
\theta_{i j}<\theta_{i k}
$$

that is, the cumulative probability of an odd number of cross-overs, and therefore that of the average number of cross-overs as well, as a function of coordinates expressed relative to a given origin, is continuous and monotonically increasing. Then, with (1) we have: 


$$
\begin{cases}\text { (i) } \theta_{i j} \geqq 0 & \text { for all } i, j=1, \ldots, n \\ \text { (ii) } \theta_{j i}=\theta_{i j} & \text { with equality iff } i=j \\ \text { (iii) } \theta_{i k} \leqq \theta_{i j}+\theta_{j k} & \text { for all } i, j=1, \ldots, n \\ \text { for } i, j, k=1, \ldots, n\end{cases}
$$

There will be equality in (iii) only if $k=i$ or $k=j$, or if there is complete positive interference and $L_{j}$ lies between $L_{i}$ and $L_{k}$. Hence $\theta_{i j}$ satisfies the metric axioms, although it is not an Euclidean metric.

\section{(ii) Non-parametric mapping from recombination data}

As no functional relationship can be given between map distances $\left\{d_{i j}\right\}$ and recombination fractions $\left\{\theta_{i j}\right\}$, a non-parametric method must be used, based on order relations. From (3), (4) and (5), it appears that the mapping problem consists in finding coordinates $\left\{x_{i}\right\}$ satisfying (3) such that

$$
\begin{cases}\text { whenever } & \text { (i) } \theta_{i j} \leqq \theta_{i k} \text { and } x_{i}<x_{j}<x_{k} \\ \text { then } & \text { (ii) } d_{i j} \leqq d_{i k}\end{cases}
$$

or, equivalently:

$$
\begin{cases}\text { whenever } & \text { (i) } \theta_{i k} \leqq \theta_{i j}+\theta_{j k} \\ \text { then } & \text { (ii) } d_{i k} \leqq d_{i j}+d_{j k}\end{cases}
$$

with equality in (ii) when $x_{i}<x_{j}<x_{k}$, all coordinates being expressed relative to the same origin and being assumed non-negative. But (6) or (7) cannot yield a simple algorithm for solution because: order being unknown, all possible orders should be tried; (7.i) cannot be checked in many cases because of missing values. Moreover, $\left\{\theta_{i j}\right\}$ are not known, but estimated by $\left\{y_{i j}\right\}$ with errors $\left\{s_{i j}\right\}$.

An approximation to this problem consists in finding $\left\{x_{i}\right\}$ satisfying (3) such that:

$$
\begin{cases}\text { whenever } & \text { (i) } y_{i j} \leqq y_{k l} \\ \text { then, as much as possible, } & \text { (ii) } d_{i j} \leqq d_{k l}\end{cases}
$$

these constraints being applied only to the available $\left\{y_{i j}\right\}$. Coordinates $\left\{x_{i}\right\}$ will be found by minimising some loss-function. Estimates $\left\{y_{i j}\right\}$ should be weighted according to their precision as well as the information they provide on distances between loci. Although eq. 8 would hold exactly if recombination values were estimated without error and interference were uniform, recombination estimates $\left\{y_{i j}\right\}$ and map distances $\left\{d_{i j}\right\}$ may not be perfectly monotonic because of fluctuations of estimates around their expectations or variable interference along the chromosome.

\section{(iii) Guttman's algorithm for solution}

The problem specified by (8) and (3) is the restriction to one dimension of the more general non-parametric multidimensional scaling problem. The first attempt toward a solution was presented by Shepard (1962a,b); more formal definitions and algorithms for solution are principally due to Kruskal $(1964 a, b)$ and Guttman (1968). The present problem seems an ideal case of application in that the underlying "spatial structure" and its dimensionality are not hypothesised, but known to exist. Our experience with this topic (Lalouel, 1973, 1975) has led us to adopt the general algorithm $38 / 1-\mathrm{E}$ 
presented by Guttman (1968) with extensions that will be mentioned below, and we wrote the computer program GENMAP for the CDC 3100 computer as a solution of the mapping problem. To avoid technical details that might obscure the subject-matter of this paper, the following presentation will be restricted to the considerations that are necessary for an understanding of the method and a discussion of the results, details being available in the literature cited.

To render the criterion (8) operational, a loss function must be defined. In this we follow Guttman who defines the coefficient of monotonicity:

$$
\mu=\sum_{i j} e_{i j} d_{i j} \hat{d}_{i j} /\left[\left(\sum_{i j} e_{i j} d_{i j}^{2}\right)\left(\sum_{i j} e_{i j} \hat{d}_{i j}^{2}\right)\right]^{\frac{1}{2}}
$$

and the alienation:

$$
a=\left(1-\mu^{2}\right)^{\frac{1}{2}} \text {. }
$$

$\left\{e_{i j}\right\}$ are weights to be defined further. Elements $\left\{\hat{d}_{i j}\right\}$, called " rank images", are permutations of the $\left\{d_{i j}\right\}$ which reflect the discrepancies between the sets $\left\{d_{i j}\right\}$ and $\left\{y_{i j}\right\}$ when each set is arranged in order of increasing values. The algorithm used in GENMAP to maximise $\mu$ corresponds to Guttman's scmi-strong monotonicity condition, which best allows for the treatment of tied values. Map coordinates $\left\{x_{i}\right\}$, and consequently map distances $\left\{d_{i j}\right\}$, will be obtained by maximising $\mu$, or equivalently minimising $a$.

However, from convergence considerations, we adopted Guttman's (1968) two-phase iterative algorithm which minimises:

$$
z=\frac{1}{4} \sum_{i j} e_{i j}\left(d_{i j}-\hat{d}_{i j}\right)^{2}
$$

whose gradient is

$$
\partial z / \partial x_{i}=\sum_{j} e_{i, j}\left[\left(x_{i}-x_{j}\right)-\hat{d}_{i j} \sigma_{i j}\right]
$$

where $\sigma_{i j}$ is $\operatorname{sgn}\left\{\left(x_{i}-x_{j}\right)\right\}$, i.e. unity with the sign of this difference, rather than maximising $\mu$ directly. The minimum of $z$ corresponds to the maximum of $\mu$, provided that the $\left\{d_{i, j}\right\}$ are normed when there are missing values.

Briefly, the proccdure is as follows: given the coordinates $\left\{x_{i}\right\}$ of $n$ loci on the genetic map at some iteration $s$, distances $\left\{d_{i j}^{(s)}\right\}$ are computed, using (3), with rank images $\left\{\hat{d}_{i, j}^{(s)}\right\}$, making use of the ranking of the $\left\{y_{i j}\right\}$ arranged in order of increasing value. $z^{(s)}$ is computed following (11), and a new solution $\left\{x_{i}^{(s+1)}\right\}$ is obtained by minimising $z^{(s)}$, within iteration $s$, assuming the $\left\{\hat{d}_{i j}^{(s)}\right\}$ to be known quantities within s. Writing the gradient of $z^{(s)}$ and setting its derivatives to zero yields the algorithm:

$$
x_{i}^{(s, t+1)}=\left(\sum_{j} e_{i j}\right)^{-1}\left[\sum_{j} e_{i j} x_{j}^{(s, t)}+\sum_{j} e_{i j} \hat{d}_{i j}^{(s)} \sigma_{i j}^{(s, t)}\right]
$$

$t$ indicating subitcrations performed within s. Rather than requiring complete convergence of (13), only 10 subiterations are performed, as suggested by Guttman (1968), hence $x_{i}^{(s+1,0)}=x_{i}^{(s, 10)}$ and the procedure is repeated until convergence is reached.

As with any iterative minimisation procedure, an initial set of trial values $\left\{x_{i}^{(0)}\right\}$ is required. Guttman (1968) proposes a method to obtain 
an initial approximation which is satisfactory when there is no missing value, but is ineffective for the mapping problem, as generally many values are missing. Hence, an empirical set of trial values has to be determined. Another difficulty of such minimisation procedures is that of obtaining the absolute minimum. Convergence to this absolute minimum is guaranteed by Guttman's two-phase algorithm when there are no missing values, but this is no longer true when many values are missing. To avoid taking a local minimum as a solution, our program GENMAP prints detailed outputs such as deviations $\left\{d_{i j}-\hat{d}_{i j}\right\}$, playing a central role in the gradient of $z$, the elements of the gradient itself $\left\{\partial z / \partial x_{i}\right\}$, and partial alienations for each locus, defined as $a_{i}=\left(1-\mu_{i}^{2}\right)^{\frac{1}{2}}$, where $\mu_{i}$ is given by (9) with summations restricted to index $j$. Alternate orders may be tried for closely mapped loci in order to ensure that the absolute minimum has been reached.

It seems worth while to us to close this technical discussion by stressing the fact that recombination estimates determine the solution only through their ordering. It follows that any monotonic transformation of the $\left\{y_{i j}\right\}$, such as any of the mapping functions referred to above, will lead to identical solutions with this non-parametric method. Another consequence of using a non-parametric approach is that the solution $\left\{x_{i}\right\}$ it yields is scale-free. However, scaling of the $\left\{x_{i}\right\}$ could be done, following Bridges and Morgan (1919, 1923), by taking $d_{i j}=y_{i j}$ for that particular, small recombination estimate considered the most reliable. Alternately, since for recombination values smaller than $0 \cdot 10$, the equality $d_{i j}=\theta_{i j}$ holds to a good degree of approximation, scaling has been done here by computing the regression coefficient $l=\Sigma e_{i j} y_{i j} d_{i j} / \Sigma e_{i j} d_{i j}^{2}$, summation being restricted to recombination estimates smaller than $0 \cdot 10$, and expressing subsequently map distances as $d_{i j}^{*}=l d_{i j}$.

\section{(iv) Weights associated with recombination estimates}

Information provided on separation between loci varies inversely with recombination $\theta_{i j}$, that is we should have $e_{i j}=0$ when $\theta_{i j}=0.5$; moreover, weights should be functions of the precisions of the estimates $\left\{y_{i j}\right\}$. Considering the form of the function $z$ as expressed by (11), in the applications to follow the weight associated to an estimate $y_{i j}$, with standard error $s_{i j}$, has been taken as the reciprocal of the variance of the Kosambi transform of the estimate $y_{i j}$, that is:

$$
\begin{aligned}
e_{i j} & =\left(1-4 y_{i j}^{2}\right)^{2} / s_{i j}^{2}, & & \left(y_{i j}<0 \cdot 5\right) \\
& =0 & & \left(y_{i j} \geqq 0 \cdot 5\right)
\end{aligned}
$$

It is likely that improvement of the method could be obtained by a more rigorous definition of these weights, although probably not affecting very much the final solution obtained.

\section{(v) "Neuterised" map when data from both sexes are used}

When data are available separately on each sex, they should be used separately to yield genetic maps in the event of significant sex differences. However, in such a case, one may possibly be interested in deriving a unique (neuterised) genetic map that would allow to predict recombinations in crosses with sex unknown. Rather than averaging estimates among 
sexes, a procedure which might destroy order relationship when intcrference varics according to physical location as well as sex, the present method may be extended so that, deriving rank images $\left\{\hat{d}_{i j a}\right\},(a=1,2)$ separately from each sex, $z$ becomes:

$$
z=\frac{1}{4} \sum_{i j a} e_{i j a}\left(d_{i j}-\hat{d}_{i j a}\right)^{2},
$$

and the algorithm is modified accordingly.

4. Application to bridges and morgan's (1923) data on chromosome 3 OF DROSOPHILA

\section{(i) The data}

We present now an application of this non-pararnetric mapping method to data on chromosome 3 of Drosophila melanogaster as, probably because they were published later, Bridges and Morgan's (1923) data for this chromosome are more extensive than those of their earlier studies of other chromosomes. We are not aware of more complete recombination data presented in a single report. Later data are widespread in the literature but of difficult access, and often incompletely represented. For a preliminary study such as the present one, this seemed to us a body of data important enough.

We wrote our program GENMAP simply so as to make the procedure fast, and as a consequence limiting the maximum number of loci that can be handled simultaneously to 25 (however, this limitation could be casily relaxed). The mutants are distributed by Bridgcs and Morgan into classes in order of their practical value, rated in terms of their ease of recognition, viability, and scatter of estimates among replicate crosses. We consequently retained all loci evaluated as first class, the complement to 25 being selected by retaining those loci that had the largest numbers of recombination estimates available. Many of these estimates have been obtained in multipoint test-crosses and the data are separated by Bridges and Morgan into two categories, primary and secondary data, in an attempt to reduce the dependencies arising from simultaneous estimations from a single cross. However, because many estimates presented for both categories are themselves simple averages of estimates occurring from various experiments, and in view of othcr approximations, this refinement seemed to us unnecessary for mapping, and consequently we used Bridges and Morgan's total data (1923, table 2, pp. 4-6). These data are reproduced in tablc 1 .

\section{(ii) Genetic map obtained by non-parametric mapping}

Variances of estimates $\left\{y_{i j}\right\}$ are needcd to compute weights $\left\{e_{i j}\right\}$ according to (14). However, rather than variances of estimates, Bridges and Morgan (1923) give sample sizes, which are often pooled over various experiments. In the following, we took as variance of an estimate $y_{i j}$ the binomial variance $y_{i j}\left(1-y_{i j}\right) / \mathcal{N}_{i j}$, where $\mathcal{N}_{i j}$ is the total number of flies for which recombination between $L_{i}$ and $L_{j}$ was studied. This certainly is a crude approximation, and for a definitive mapping, efficient estimates based on various experiments should be derived, so that the variance would reflect variability between experiments (for example, see Finney, 1949). When various 


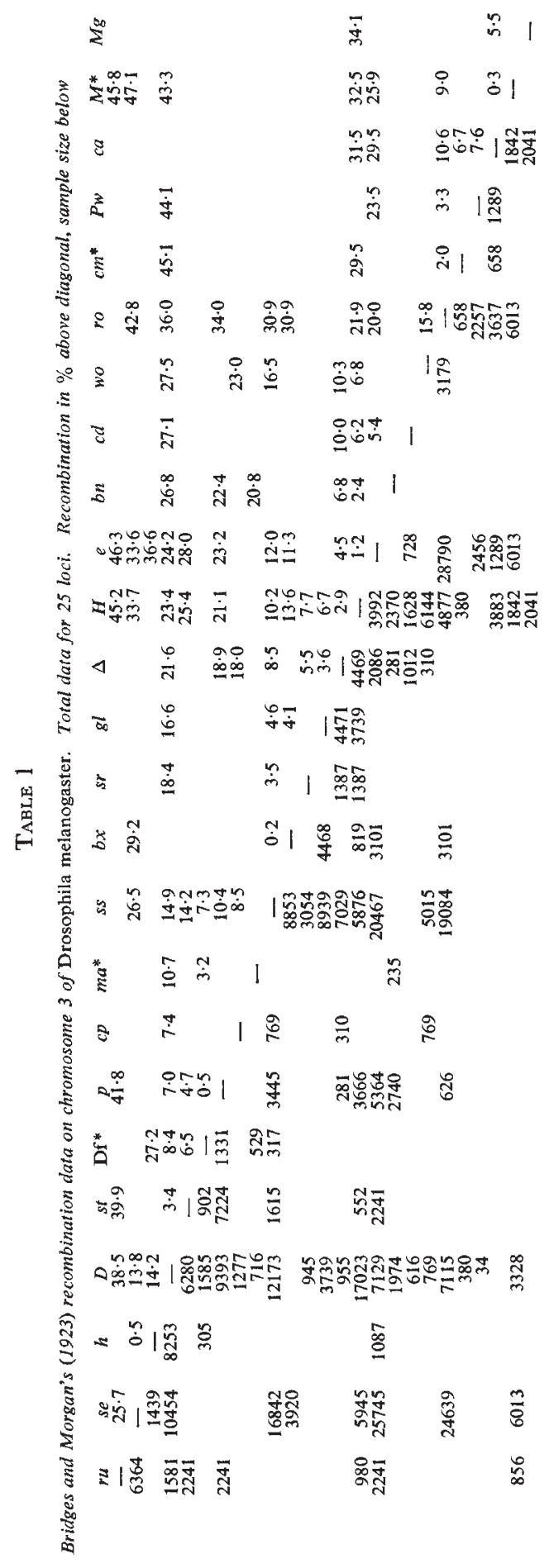

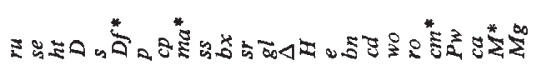


experimental designs have been used, this would require maximum likelihood scoring, possibly allowing for differential viability and incomplete penetrance.

Initial coordinates of loci $\left\{x_{i}^{(0)}\right\}$ were taken as the order given by Bridges and Morgan (1923, p. 27), with equidistant intervals. However, four loci (namely $D f, m a, c m$ and $M$ ) fitted poorly, as attested by large partial alienations $(>0.15$, whereas the total alicnation was 0.095) and important inconsistencies of estimates, and affected the mapping of neighbouring loci. That the data for these loci are less reliable is confirmed by their belonging to valuation classes 2 or 3 , and important variability of some estimates in replicate experiments. They were consequently deleted for this analysis, but could be reintroduced at a later stage, once a solution has been found for the remaining loci.

In fig. 1, we present simultaneously the map given by Bridges and Morgan and the map obtained by the non-parametric method, for the
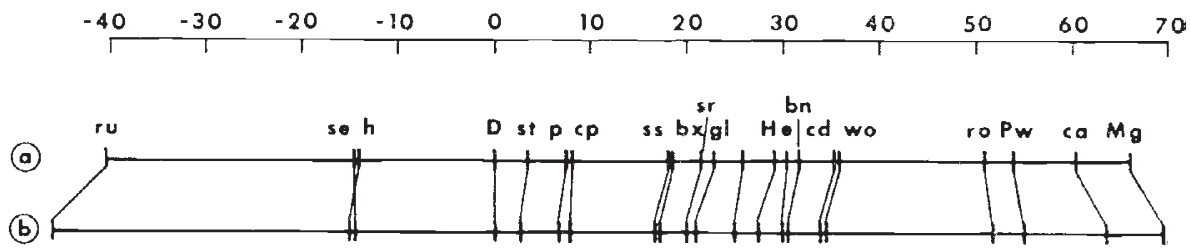

FIG. 1.-Mapping of chromosome 3 of Drosophila melanogaster. Data are from Bridges and Morgan (1923). (a) Bridges and Morgan (1923) map, alicnation $=0 \cdot 108$; (b) nonparametric map, alienation $=0 \cdot 065$.

remaining 21 loci. Locus $D$ (Dichaete) is taken as the origin because, according to Bridges and Morgan, it is the most reliable mutant and the one for which the largest number of recombination values are available (on the contrary, data for distal loci $r u$ and $M g$ are scanty). Coordinates and partial alienations for each locus are given in table 2. As previously dcfined, partial alienation for a given locus $L_{i}$ rcflccts both the degree of resolution of possible inconsistencies of estimates $y_{i j}$ and the extent to which variable interference, particularly on each side of the locus, has influcnced the solution.

TABLE 2

Non-parametric mapping of chromosome 3 of Drosophila melanogaster

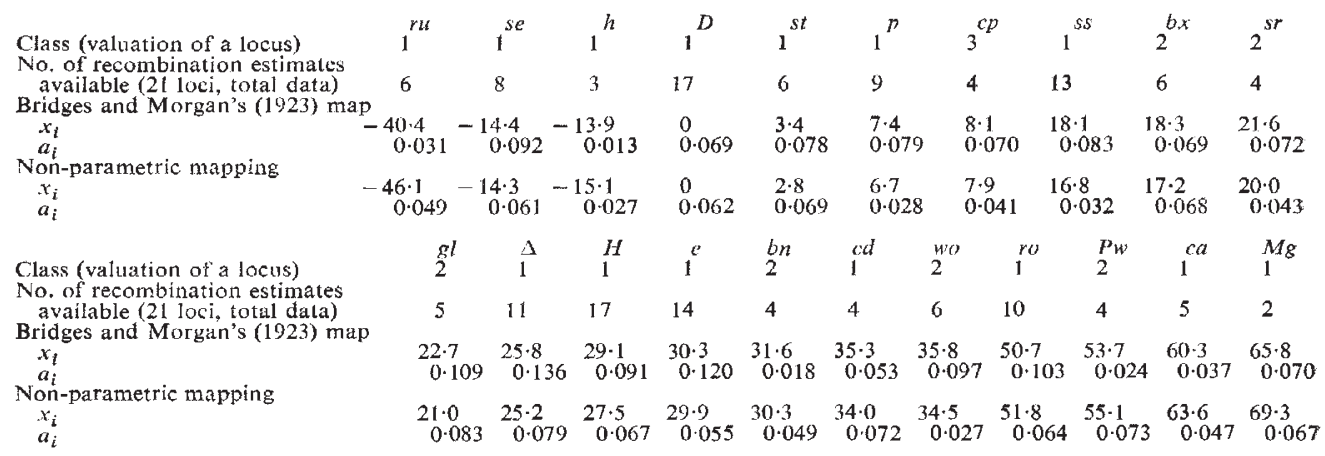


Order of loci given by both maps is the same, except for the two loci $s e, h$, which are so close $\left(y_{s e, h}=0.005\right)$ that their relative ordering is probably beyond the power of resolution of the present method. However, the order $h-s e-D$ given by the non-parametric method is consistent with the data that determine it $\left(y_{h, D}=0.142 ; y_{s e}, D=0.138\right)$, as can be seen in table 1 . The alternate order given by Bridges and Morgan stems from considerations of other data on three-point tests leading to estimates $y_{h, D}<y_{s e, D}$ (Bridges and Morgan, 1923, p. 15). With regard to the very different approaches followed to get those two maps, general agreement is impressive for most of the chromosome: genetic distance between se and ro differs by a mere centimorgan. That terminal loci $r u, P w, c a$ and $M g$ are probably less reliably located (presumably in both maps) can be explained by the nature of the data available. $M g$ is mapped from only two estimates $(0.055$ with $c a, 0.341$ with $H)$, obviously insufficient to deal with interference. Relationships of $c a$ and $P w$ with their neighbours are probably poorly documented by the available estimates. As for $r u$, its distal location together with the nature of the data available (the two smallest estimates available are $y_{r u, s e}=0.257$ and $y_{r u, D}=0.385$ ) makes its mapping very unreliable; with the present method, the only constraint on the segment ru-se is that it be sensibly equal to the segment se-ss. If interference is different in these two segments, map distance ru-se will be affected (that this is the case will be seen later). Bridges and Morgan located $r u$ by computing coincidence for $r u-s e-D$, which is close to zero. Data on Henna (van Atta, 1932), on the left of se, also suggest almost complete interference on ru-se; there is no doubt that data available on $r u$ are not satisfactory for mapping, and that data on loci between $r u$ and se would allow a much better determination of this segment.

\section{(iii) Recovery of an approximate mapping function}

An interesting consequence of this non-parametric approach is that, as no functional relationship is assumed between recombination and map distance in the mapping process, one can attempt to recover a mapping function that would hold approximately over the whole range of the region that has been mapped, a problem clearly specified by Owen (1950, p. 130).

Rather than examining which, of various mapping functions, would best apply to the present data, we used the general mapping function (Rao, Morton, Lindsten, Huttén, Yee, in preparation):

$$
\begin{aligned}
& w=\left[p(2 p-1)(1-4 p) \ln (1-2 \theta)+16 p(p-1)(2 p-1) \tan ^{-1}(2 \theta)\right. \\
& \left.\quad+2 p(1-p)(8 p+2) \tanh ^{-1}(20)+6(1-p)(1-2 p)(1-4 p) \theta\right] / 6,
\end{aligned}
$$

where $p$, to be estimated, reflects the average intensity of interference over the region under study. This function yields Haldane's (1919) mapping function when $p=1$ (no interference), Kosambi's (1944) when $p=1 / 2$, Carter and Falconer's (1951) when $p=1 / 4$ and $w=\theta$ when $p=0$ (complete interference). Any map distance, $d_{i j}$, of fig. 1 being in general constrained, in a non-parametric manner, by several recombination estimates, errors on $\left\{d_{i j}\right\}$ can be considered negligible relative to errors of estimates $\left\{y_{i j}\right\}$. An estimate of $p$ over the whole region was computed by minimising

$$
f=\Sigma e_{i j}\left(d_{i j}-w\left(y_{i j}\right)\right)^{2} / \Sigma e_{i j} d_{i j}^{2}
$$


where $e_{i j}$ is defined by (14) and the summation concerns only the estimates $\left\{y_{i j}\right\}$ available that are greater than $0 \cdot 10$. This yielded an estimate of $p=0.56$ with $f=0.004$, and. as can be seen in fig. 2 , this seems to provide a fairly good fit. Taking equal weights $\left\{e_{i j}\right\}$ in (17) gave an estimate of $p=0.45(f=0.008)$. This indicates that, for these data, interference is, on the average, at Kosambi level.

\section{(iv) Non-uniformity of interference}

We consider interference from a purely genetic point of view; that is, for two contiguous segments denoted 1 and 2 , rewriting (1), we have:

$$
\theta_{1+2}=\theta_{1}+\theta_{2}-2 C_{1,2} \theta_{1} \theta_{2}
$$

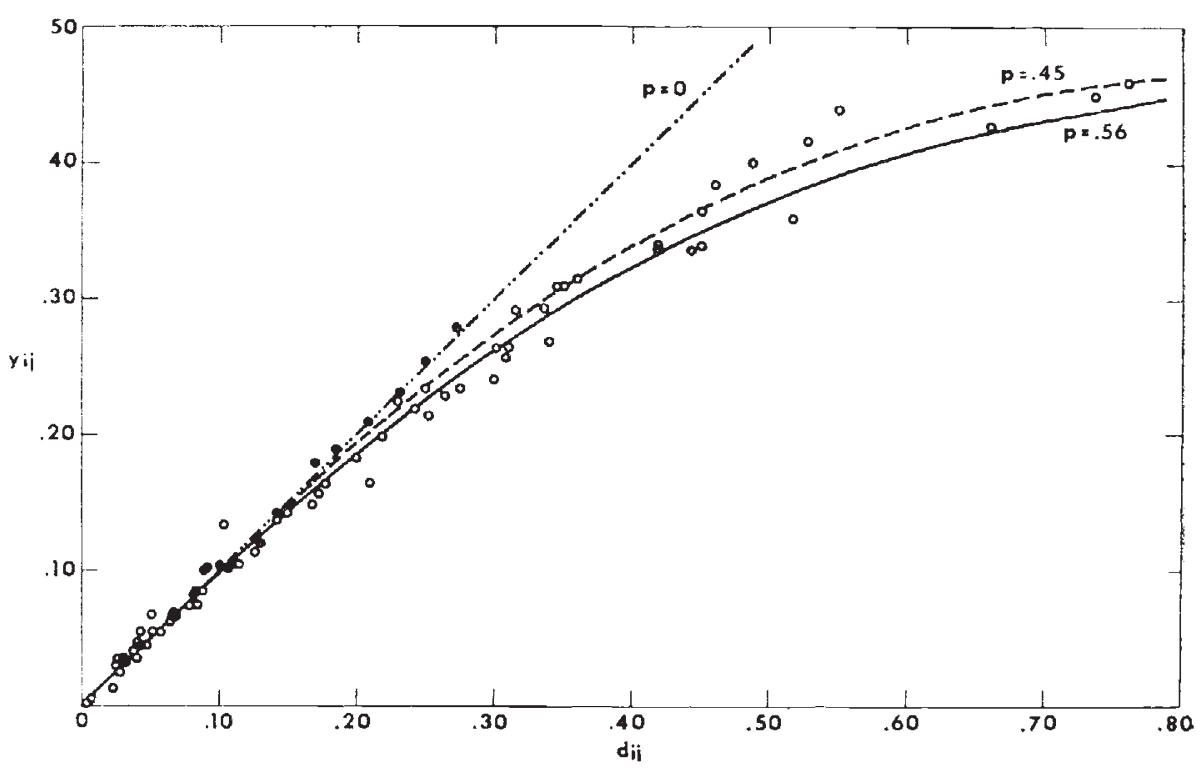

FIG. 2.- Recovery of an approximate mapping function, by fitting map distances obtained by the non-parametric method to the Rao et al. (in preparation) generalised mapping function. Solid curve: differential weights used; broken curve: equal weights used. Solid dots are $\left(d_{i j}, y_{i j}\right)$ values for segments contained in the st-e region.

where the coincidence coefficient $C_{1,2}=z_{1,2} / \theta_{1} \theta_{2}$ is the ratio of the probability of simultaneous recombination in both segments to that expected under independence of recombinational events in each of the two segments. Consider two other contiguous segments 3,4 located in a different part of the chromosome and such that $\theta_{1}=\theta_{3}, \theta_{2}=\theta_{4}$. Then, if and only if interference is uniform along the genetic map, $C_{1,2}=C_{3,4}$, hence $\theta_{1+2}=\theta_{3+4}$, implying $w_{1+2}=w_{3+4}$. However, coincidence being defined for two segments (hence for three loci if contiguous, for four if not contiguous), it is difficult to establish a relation between variation of coincidence and variation of interference on the genetic map. Anderson and Rhoades' (1931) index of intensity of interference $I$ or Owen's (1950) Kosambi's coefficient $K$ attempt to alleviate this by making these coefficients indicators of the 
intensity of interference on one segment, although still only approximately. Various studies suggest that interference is not uniform along the genetic map (see for example Anderson and Rhoades, 1931), although this might not be true on the cytological map, as argued by Mather (1936). Whether interference is really an interaction between occurrences of chiasma or a consequence of the non-uniformity of the chiasma distribution is not relevant to the question of generating a genetic map from recombination data. As stated earlier, a method allowing for variable interference should in general associate with equal recombination values $\theta_{i j}=\theta_{k l}$, map distances $w_{i j}+w_{k l}$, or equivalently reflect the importance of deviation from additivity in (18), of which coincidence is an index.

That interference might vary along the genetic map and that this is reflected in the non-parametric map of fig. 1 is suggested by examining how the deviations from the fit of the function (16) for $p=0.56$ are distributed along the chromosome. It then appears that most of the points $\left(y_{i j}, d_{i j}\right)$ corresponding to recombination values larger than $0 \cdot 10$ and pertaining to loci within the segment $(s t, e)$ are found near the line $w=\theta$ $(p=0)$, as can be seen in fig. 2. This led us to investigate this question in more detail. Coincidences values between contiguous segments were computed wherever available data permitted it, assuming independence of estimates, such that:

$$
C_{1,2}=\left(y_{1}+y_{2}-y_{1+2}\right) / 2 y_{1} y_{2}
$$

with large sample variance

$\operatorname{var}\left(C_{1,2}\right)=\left[y_{2}^{2}\left(y_{1+2}-y_{2}\right)^{2} \sigma_{1}^{2}+y_{1}^{2}\left(y_{1+2}-y_{1}\right)^{2} \sigma_{2}^{2}+y_{1}^{2} y_{2}^{2} \sigma_{1+2}^{2}\right] / 4 y_{1}^{4} y_{2}^{4}$,

obviously an approximation in view of the way recombination estimates were obtained. On the other hand, for all recombination value $y_{i j}$ available, we computed an estimate of the intensity of interference $p_{i j}$, using (16) iteratively so as to express $p_{i j}$ as a function of $y_{i j}$ and $d_{i j}$. Because each coordinate $x_{i}$ is constrained by many recombination values, one can assume errors on $\left\{x_{i}\right\}$ negligible, so that errors in any $p_{i j}$, computed as such, mainly depend on error in $y_{i j}$, at least for that part of the map (from se to $w o$ ) for which many recombination values were available. A summary is given in table 3 , where some representative values of $C_{i k j}$ and $p_{i j}$ are reported. Comparisons are difficult because whereas coincidence is a characteristic of two segments, $p$ is defined for one segment. However, this seems to suggest: (1) that there is negative genetical interference across the centromere (located between loci st and $p$ ), at least when loci followed are located in its vicinity; (2) interference may be complete, at least on the right arm (data on the left arm are insufficient), from the centromere up to (approximately) the locus $\Delta$; (3) interference seems to decrease beyond that region; (4) whether it rises again in the distal part of the right arm cannot be determined due to insufficient data; (5) the $p$-values computed seem to reflect the same trend ( $p_{D, p}$ excepted). We take this as a suggestive evidence of non-uniformity of interference along the chromosome, and that this variable interference is, at least in part, reflected by the non-parametric mapping. More experience with this method, particularly with simulated data assuming variable interference, is required to confirm or refute these suggestions. 
TABLF, 3

Some representative values of coincidence and interference parameter $\mathrm{p}$ for segments of the non-parametric map

\begin{tabular}{|c|c|c|c|c|}
\hline$i$ & $(k)$ & $j$ & $C_{i(k) j} \pm \sigma$ & $P_{i_{j}}$ \\
\hline$D$ & $(s t)$ & $p$ & $3 \cdot 44 \pm 1 \cdot 13$ & $<0$ \\
\hline$D$ & $(s t)$ & ss & $2 \cdot 80 \pm 0 \cdot 80$ & \\
\hline$D$ & $(p)$ & ss & $1.72 \pm 0.37$ & 85 \\
\hline$D$ & $(p)$ & $\Delta$ & $1.63 \pm 0.85$ & \\
\hline$D$ & $(s s)$ & $\Delta$ & $0.71 \pm 0.55$ & \\
\hline$p$ & $(s s)$ & $\Delta$ & $0 \pm 1.37$ & $=0$ \\
\hline$p$ & (ss) & $H$ & $-2 \cdot 36 \pm 0.45$ & $<0$ \\
\hline ss & $(\Delta)$ & wo & $1.31 \pm 0.83$ & \\
\hline ss & $(H)$ & $w o$ & $0.36 \pm 0.51$ & 0 \\
\hline$D$ & $(s s)$ & wo & $0.79 \pm 0.34$ & \\
\hline$D$ & $(\Delta)$ & 200 & $0.99 \pm 0.49$ & $\cdot 70$ \\
\hline$D$ & $(H)$ & wo & $0 \cdot 85 \pm 0.52$ & \\
\hline se & $(D)$ & $H$ & $0.54 \pm 0 \cdot 11$ & $\cdot 55$ \\
\hline$s e$ & $(s s)^{\prime}$ & $H$ & $0.55 \pm 0.14$ & \\
\hline
\end{tabular}

\section{Discuussion}

This study shows that it is possible to generate a genetic map from pairwise recombination data without having to assume a (generally unknown) mapping function. However, it also raiscd interesting questions related to the mapping problem, that deserve particular attention.

Several of these questions, namely interference across the centromere, variable interference on each arm, and the meaning of coincidence are very much interrelated. That coincidence varies not only according to the total segment of a chromosome considered but also with its relative location with respect to the centromere has been amply recognised (see for example Bridges and Morgan (1919), (1923); Owen (1948), quoted in Owen (1950)), suggesting that genetic interference, on one arm of chromosomes of sufficient length for multiple cross-overs to occur, is high in the neighbourhood of the centromere, decreases in the mid-region, and may rise again toward the distal end. An explanation of this phenomenon would require a better knowledge of the mechanism of crossing-over; however, it is worth noting that, under Mather's theory of localised chiasma, complete genetic interfcrence in the proximal region could result from the absence of multiple cross-overs in that region (for example, see Carter (1954), fig. 3, p. 214).

The debate over the existence of interference across the centromere has been confused by misinterpretations of coincidence values as well as the possibility that there might not be a unique answer for any chromosome of any species. In developing his theory on chiasma formation, Mather (1936) recapitulated evidence, both genetical and cytological, demonstrating independence of the two arms of a chromosome. However, as seen in table 3 , coincidence greater than one was found with the present data when two of the loci studied are on different arms but close to the centromere. Although the significance of these results cannot be evaluated, corroborative evidence for the third chromosome of Drosophila can be found in Kikkawa (1935, table 1), Pätau (1941), or the data on D-si-ss of Clausen and Collins (1922). Coincidence valucs in excess of one have been con- 
sidered as evidence of negative chiasma interference, that is the occurrence of one chiasma in one segment would favour the occurrence of a second chiasma in its vicinity. But in the present case, alternate explanations might be offered, preserving an hypothesis of independence of occurrence of chiasma on different arms of a chromosome. One would be to consider that, although there is no chiasma interference, chromatid interference exists across the centromere, strong in its neighbourhood and decreasing rapidly with increasing distance from the centromere. For two contiguous segments 1 and 2 separated by the centromere and short enough so as one can assume only one cross-over can occur in each segment with probabilities $p_{1}$ and $p_{2}$ respectively, coincidence will be as high as $C_{1,2}=2 / p_{1} p_{2}$ in the extreme case where cross-over in each segment, when occurring simultaneously, affect the same two strands in an obligate manner. Another possible explanation was provided by Kikkawa (1935): if a region including the centromere fails to enter a state of synapsis, then coincidence greater than 1 can be observed, even in the absence of chiasma interference; in the simplified situation where one assumes that asynapsis occurs with frequence $i$, coincidence in that region will be $C=1 /(1-i)$, as shown by Kikkawa (1935). That Dichaete was found to be a small inversion (Morgan, Bridges, and Schultz, 1937) raised the possibility that this could be the cause of most coincidences found greater than one, but it seems (see Kikkawa, 1935), that similar observations were found in crosses not involving Dichaete.

Whatever the real answer to that question, the preceding discussion emphasises that a cytological demonstration of the independence of occurrence of chiasmas in different arms will not attest to the absence of a possible genetical interference across the centromere; conversely, estimation of coincidence, one segment including the centromere, significantly greater or smaller than one cannot be in general taken as a demonstration of nonindependence of each arm. This is so because coincidence is not a property of a segment, but of two segments, and the following example may be useful: consider seven loci $a, b, \ldots, g$ in that order, locus $d$ being undistinguishable from the centromere, and such that $\theta_{a b}=\theta_{b c}=\ldots=\theta_{f g}=$ $0 \cdot 10$; assume independence of each arm, complete synapsis, and no chromatid interference; assume moreover that there is complete interference in the two regions $b-d$ and $d-f$, and no interference elsewhere. Then $C_{b c d}=$ $C_{d e f}=0, C_{a d f}=C_{b d f}=C_{c d f}=1$, but $C_{a e f}=0 \cdot 84, C_{b e f}=0 \cdot 77, C_{c e f}=0.56$. That Kosambi coefficient is not a more satisfactory measure of interference can be seen from the fact that $C_{a e f} \neq C_{a d f}$. To study genetical interference across the centromere, it is necessary to take as intermediate locus one that is in the immediate vicinity of the centromere.

These remarks have important consequences for the mapping problem. If interference were complete or almost complete in proximal regions but recombinational events in the two arms were indcpendent, then a mapping of the whole chromosome, using a mapping function of the form (16), would necessarily be approximate in proximal regions, for it would attempt to reconcile these two phenomena. If any such mapping function were used, a more accurate map would necessitate having one locus $c$ known to be in the immediate vicinity of the centromere and mapping each arm separately. Prediction of the recombination valuc between two loci $a, b$ on different arms would be obtained by reading map distances $d_{a, c}$ and $d_{c, b}$, applying an inverse mapping function, and computing the recombination 
$y_{a b}$ from (1), assuming independencc. However, the results presented in table $3 \mathrm{sccm}$ to indicate that the non-parametric method behaves satisfactorily in such a situation.

A last point descrving attention concerns the planning of experiments most appropriate for genetic mapping. The data assembled for such a procedure must satisfy several conditions, some of which are clearly specified by Bridges and Morgan (1923): one should select mutants having good "laboratory" properties, such as good viability, complete penetrance, easy recognition, and so on; they should be regularly spaced along the whole region to be mapped; as this requisite often cannot be satisfied for a preliminary study, this suggests that, wherever too long segments emerge with few loci, intervening loci should be added to generate a new, more accurate map. Other requirements arise with the present method. For the weighting suggested here by formula (14) to be satisfactory, sample sizes should be of the same magnitude, or at least not functions of recombination; that is, if sample sizes were systematically larger for large recombinations, the present weighting would be inappropriatc and interference unsatisfactorily reflected in the map. Large recombination values, at least for Drosophila, carry little information on separation and may not be necessary altogether. As for the design of the crosses to be carried out to estimate recombination values, it might well be that two-points tests, in the form of the two reciprocal balanced backcrosses, could be more appropriate for the purpose of mapping (if not of detection of linkage or rcsolution of very close sequences) than multipoint tests crosses, where dependencies are such and balancing so often impossible that one might suspect their sensitivity to viability, penetrance, or other uncontrolled effects. Whether more or less effort would be required in using two-points tests is a question whose answer is with the experimenters.

Acknozeledgments. - This investigation was suggested to us by Newton E. Morton. We gladly acknowledge his continuing interest with this work. Discussions of this topic with C. J. Maclean and D. C. Rao were also particularly helpful.

\section{REFERENCES}

ANDERSON, F. G., AND RHOADEs, M. M. 1931. The distribution of interference in the Xchromosome of Drosophila. Pap. Mich. Acad. Sci., 13, 227-233.

BAILEY, N. T. J. 1961. Introduction to the Mathemalical Theory of Genetic Linkage. Oxford University Press, London.

BRIDGES, C. B., AND MORGAN, T. H. 1919. Contribution to the genetics of Drosophila melanogaster. II. The second-chromosome group of mutant characters. Carnegie Inst. Wash. Publ., 278, 123-304.

BRIDGES, C. B., AND MORGAN, T. H. 1923. The third-chromosome group of mutant characters of Drosophila melanogaster. Carnegie Inst. Wash. Publ., 327, 1-251.

CARTER, T. C. 1954. A search for chromatid interference in the male house mouse. $Z$. Ind. Abst. Vererb., 86, 210-223.

CARTER, T. G., AND FAlCONER, D. s. 1951. Stocks for detecting linkage in the mouse and the theory of their design. 7. Genet., 50, 307-323.

Clausen, R. E., AND Collins, J. L. 1922. The inhcritance of ski wings in Drosophila melanogaster. Genetics, 7, 385-426.

DunN, L. G., AND BENNETT, D. 1967. Sex differences in recombination of linked genes in animals. Genet, Res., 9, 211-220.

FINNEY, D. J. 1949. The estimation of the frequency of recombinations. I. Matings of known phase. 7. Genet., 49, 159-176. 
Fisher, R. A., AND landauer, w. 1953. Sex differences of crossing-over in close linkage. Amer. Nat., 87, 116.

FISHER, R. A., LYON, M. F., AND OWEN, A. R. G. 1947. The sex chromosome of the house mouse. Heredity, 1, 355-365.

Gutrman, L. 1968. A general nonmetric technique for finding the smallest coordinate space for a configuration of points. Psychometrika, 33, 469-506.

HALDANE, J. B. S. 1919. The combination of linkage values and the calculation of distance between the loci of linked factors. 7. Genet., 8, 293-303.

HALDANE, J. B. s. 1922. Sex ratio and unisexual sterility in hybrid animals. 7. Genet., 12, 101-103.

Henderson, s. A. 1961. The chromosomes of the British Tetrigidae (Orthoptera). Chromosoma, 12, 553-572.

Henderson, s. A. 1963. Chiasma distribution at diplotene in a locust. Heredity, 18, 173-190.

HENDERSON, s. A. 1969. Chromosome pairing, chiasmata and crossing-over. In Handbook of Molecular Cytology, ed. A. Lima de Faria, pp. 326-357. Oliver and Boyd, Edinburgh.

JOHn, B., AND LEWIS, K. R. 1965. The Meiotic System. Protoplasmatologia VI F1. Springer Verlag, Wien-New York.

KIKKAWA, н. 1935. Biological significance of coincidence in crossing-over. Fap. F. Genet., 11, 51-59.

Kosambi, D. D. 1944. The estimation of map distances from recombination values. Ann. Eugen., 12, 172-175.

KRUSKAL, J. B. 1964a. Multidimensional scaling by optimizing goodness of fit to a nonmetric hypothesis. Psychometrika, 29, 1-27.

KRUSKAL, J. B. 1964b. Nonmetric multidimensional scaling: a numerical method. Psychometrika, 29, 115-129.

LAlouel, J. M. 1973. Topology of population structure. In Genetic Structure of Populations, ed. N. E. Morton, pp. 139-149. University Press of Hawaii, Honolulu.

lalouel, J. M. 1975. Différenciation locale d'une population. Thése de Doctorat de Sciences, Université Paris VI, Paris.

MATHER, K. 1936. The determination of position in crossingover. I. Drosophila melanogaster. 7. Genet., 33, 207-235.

MATHER, K. 1937. The determination of position in crossingover. II. The chromosome length/chiasma frequency relation. Cytologia, Fujii. Zub. Vol., 514-526.

MATHER, K. 1938. Crossingover. Biol. Rev., 13, 252-292.

MORGAN, T. H., BRIDGES, C. B., AND SChultz, R. 1937. Constitution of the germinal material in relation to heredity. Carnegie Inst. Wash. Year Book, 36, 301.

owen, A. R. G. 1948. Ph.D. dissertation. University Library, Cambridge.

OWEN, A. R. G. 1949. The theory of genetical recombination. I. Long-chromosome arms. Proc. Roy. Soc., B, 136, 67-94.

OWEN, A. R. G. 1950. The theory of genetical recombination. Advances in Genetics, 3, 117-157.

PÄTAU, K. 1941. Cytologischer Nachweis einer positiven inteferenz über clas centromer (Der Paarungskoeffizient I). Chromosoma, 2, 36-63.

RAO, D. G., MORTON, N. E., LINDSTEN, J., HUTTÉ́N, M., AND YeE, s. 1976. A mapping function for man. In preparation.

Robinson, R. 1971. Gene Mapping in Laboratory Mammals. (2 vols.) Plenum Press, London. SHEPARD, R. N. 1962a. The analysis of proximities: multidimensional scaling with an unknown distance function. I. Psychometrika, 27, 125-140.

SHEPARD, R. N. 1962 $b$. The analysis of proximities: multidimensional scaling with an unknown distance function. II. Psychometrika, 27, 219-246.

SOKoloff, A. 1964. Sex and crossing over in Tribolium castaneum. Genetics, 50, 491-496.

VAN ATTA, E. A. 1932. Genetic and cytological studies on X-radiation induced dominant eye colors of Drosophila. Genetics, 17, 637-659.

WATsON, J. D., AND CAllaN, H. G. 1963. The form of bivalent chromosomes in newt oocytes at first metaphase of meiosis. Quart. F. Micro. Sci., 104, 281-295. 